\title{
Different Methods to Determine the Polar Cap Area
}

\author{
V. M. Mishin ${ }^{1}$, A. D. BAzARzhapov ${ }^{1}$, T. I. SAifudinova ${ }^{1}$, S. B. LunYushKin ${ }^{1}$, D. Sh. Shirapov ${ }^{1}$, \\ J. WOCH ${ }^{2}$, L. EliasSON ${ }^{2}$, H. OPGENOORTH ${ }^{3}$, and J. S. MURPHREE ${ }^{4}$ \\ ${ }^{1}$ SibIZMIR, Irkutsk, USSR \\ ${ }^{2}$ Swedish Institute of Space Physics, Kiruna, Sweden \\ ${ }^{3}$ Swedish Institute of Space Physics, Uppsala, Sweden \\ ${ }^{4}$ University of Calgary, Calgary, Canada \\ (Received January 6, 1992; Accepted May 8, 1992)
}

\begin{abstract}
The distribution of field-aligned currents (FAC) during quiet and disturbed periods is calculated with the Magnetogram Inversion Technique (MIT). The polar cap boundary is determined as the high-latitude boundary of the region 1 FAC. Statistical results on the location and size of the polar cap obtained from MIT are compared with those from two independent methods: one based on Viking auroral images and the other on mapping using the Tsyganenko 1987 magnetic field model. The three methods yield essentially the same results. The northern polar cap is shifted towards dawn or dusk, depending on the sign of the $B_{y}$ component of the interplanetary magnetic field (IMF). The polar cap area grows by a factor of 2 to 3 during substorms.
\end{abstract}

\section{Introduction}

The geomagnetic projection of the magnetospheric tail lobes are the polar caps. The plasma density is low in the lobes and, accordingly, fluxes of precipitating particles are low in the polar cap. The drop in particle fluxes or the decrease in auroral luminosity as seen in allsky camera data or satellite auroral images are observational means to directly identify the polar cap boundary. An indirect method to determine the polar cap boundary is to use the FAC distribution calculated with the magnetogram inversion technique (MIT) (MISHIN, 1990; and references therein). The high-latitude boundary of the region $1 \mathrm{FAC}$ is assumed to coincide with the polar cap boundary (e.g. COLEY, 1983; and references therein).

A few studies have been performed where polar cap boundaries were directly determined from in situ measurements (e.g. LASSEN and DANIELSEN, 1989; MENG, 1989; GUSSENHOVEN et al., 1984; MAKITA et al., 1983, 1988; MAKITA and MENG, 1984). In a study by ELPHINSTONE et al. (1990) the polar cap boundary was determined based on Viking auroral images. Recently, polar cap boundaries were also derived from the Tsyganenko model (ELPHINSTONE et al., 1991; BIRN et al., 1991). In case studies but also in a statistical study MISHIN (1990) demonstrated the applicability of the MIT method to determine the polar cap boundary.

The purpose of this paper is to compare three methods to determine the polar cap boundary, namely, methods based on Viking auroral images and the Tsyganenko magnetic field model, and the MIT method. In this paper the MIT method will be applied to a couple of the time intervals considered by ELPHINSTONE et al. $(1990,1991)$, and BIRN et al. (1991).

The significance of a reliable estimation of the polar cap boundary and the polar cap area 
is evident. The drop of the electric field potential at the polar cap boundary $\left(U_{\mathrm{pc}}\right)$ determines a necessary boundary condition in magnetospheric convection models (e.g., HAREL et al., 1981). The value of $U_{\mathrm{pc}}$ is the traditional measure of the energy and momentum transfer from the solar wind into the magnetosphere (e.g., REIFF and BURCH, 1985). The polar cap area $S$ determines the open tail magnetic flux and, consequently, the input power $\varepsilon^{\prime}$ from the solar wind. Of special importance is the information on the time variation of $S, \Psi$, and $\varepsilon^{\prime}$ in magnetospheric substorm studies (MISHIN, 1991). Observations of the polar cap boundary dynamics during varying IMF conditions have been used e.g. for tests of reconnection models (e.g., CowLEY, 1981).

The major advantage of the MIT method is its possibility to follow the polar cap dynamics continuously in time with a resolution of less than one minute over very extended time intervals. This is essential for, e.g., substorm studies and for the study of IMF-effects in the magnetosphere and the ionosphere. An apparent shortcoming is that the MIT is an indirect method depending on the assumption that the high-latitude boundary of the region $1 \mathrm{FAC}$ describes the polar cap boundary correctly. This is an obvious reason to compare MIT-results with those of more direct methods.

To illustrate the MIT-method of polar cap boundary determination some examples of FAC spatial distributions will be given in Section 2. A comparison of MIT results with those of the other methods is given in Section 3. Section 4 contains a short discussion and the conclusions.

\section{Method}

A comprehensive description of MIT is given in MISHIN (1990). Only features of the method which may be an error source in the polar cap boundary determination will be noted.

Figure 1 illustrates some examples where the polar cap boundary is determined as the high-latitude boundary of the region 1 FAC distribution obtained from MIT. It is evident that field-aligned current density distributions in the MIT data are more complex than the distributions derived by IIJIMA and POTEMRA (1976). One reason may be the averaging of measurements over long time intervals which they used. An other possible reason could be the high threshold for FAC density measurements on board satellites (for TRIAD the threshold was $2.5 \times 10^{-11} \mathrm{~A} / \mathrm{m}^{2}$; IIJIMA and POTEMRA, 1976). On the other hand, MIT may introduce some artifacts due to the harmonic functions used to approximate the geomagnetic variation field potential. These functions tend to create an additional periodicity in the FAC density distribution, i.e., artificial FAC regions. The finiteness of the series of approximating functions smooths out natural gradients and widens artificially FAC regions 1 and 2, which shifts the polar cap boundary poleward. Special computer simulations were performed to quantify these effects (MISHIN, 1990). Indeed, from these experiments it was found that the area and the radius of the polar cap decrease by factors of 2 and $2^{1 / 2}$, respectively, due to the above mentioned truncation effects of the approximating series. In the following these results are taken into account.

Another result of the above simulations is concerned with field-aligned currents inside the polar cap. It was found that there is some but only minor artificial part of MIT field-aligned current in this region. Note, that the FAC region 3 inside of the polar cap occurs in MIT-data regularly.

The next feature is that a polar cap consists of two parts created by dayside merging on 

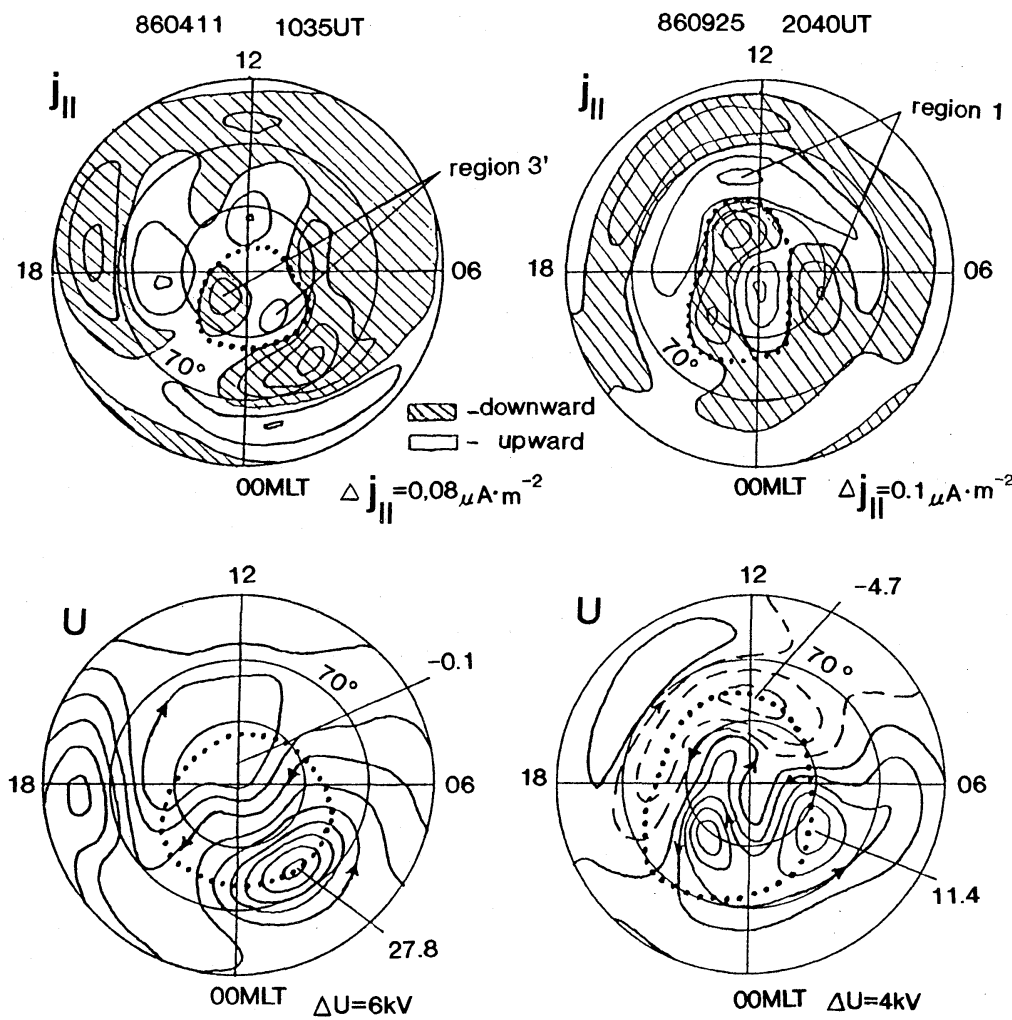

Fig. 1. Two examples to illustrate the method of polar cap boundary determination from MIT-data. (a) maps of fieldaligned current density distribution (full lines) and uncorrected polar cap boundaries (dotted lines). (b) maps of the electric potential distribution (full lines) and corrected polar cap boundaries (dotted lines).

the magnetopause and by quasi-viscous drag, respectively. Both mechanisms produce field line stretching and a polar cap which is bordered by the FAC region 1 boundary. However, there may be several (not one) so called "convection throats" (e.g. LOCKWOOD, 1990) and thus several current vortices.

It is evident from Fig. 1 that the polar cap boundary is not determined unambiguously from the MIT FAC data, alone. Therefore, as a rule, additional information from the MIT instantaneous equivalent current system and from the MIT convection system is used (for a more detailed discussion see MISHIN, 1990).

\section{Comparison with Viking Images and Mapping Results Using the Tsyganenko Model}

ELPHINSTONE et al. (1990) studied IMF-effects on the northern polar cap boundary using Viking auroral images acquired from March 1986 to January 1987. Intervals with northward directed IMF were used in their study. The IMF data were ordered according to the GSM coordinate system. The IMF data were averaged over one-hour intervals immediately preceding the image (event) considered. The events were classified into 8 groups in increments of $45^{\circ}$ of 
the IMF azimuthal angle $\beta=\tan ^{-1} B_{y} / B_{x} .65$ events (images) were selected satisfying good viewing conditions with auroral intensities higher than the dayside glow and high enough to determine a polar cap boundary. 32 events presented nearly complete views of the auroral distribution in the northern hemisphere. For each selected event the polar cap boundary was determined as the poleward boundary of the auroral luminosity in increments of 3 hour MLT. Subsequently, these values were averaged within each of the 8 groups mentioned above (according to the value of $\beta$, with steps $\Delta \beta=45^{\circ}$ ). Thus, the statistical polar cap boundary emerged for NBZ conditions when the average value of $B_{y}$ is approximately 0 . The boundaries were found by visual inspection and thus are subject to a rather high degree of uncertainty; a

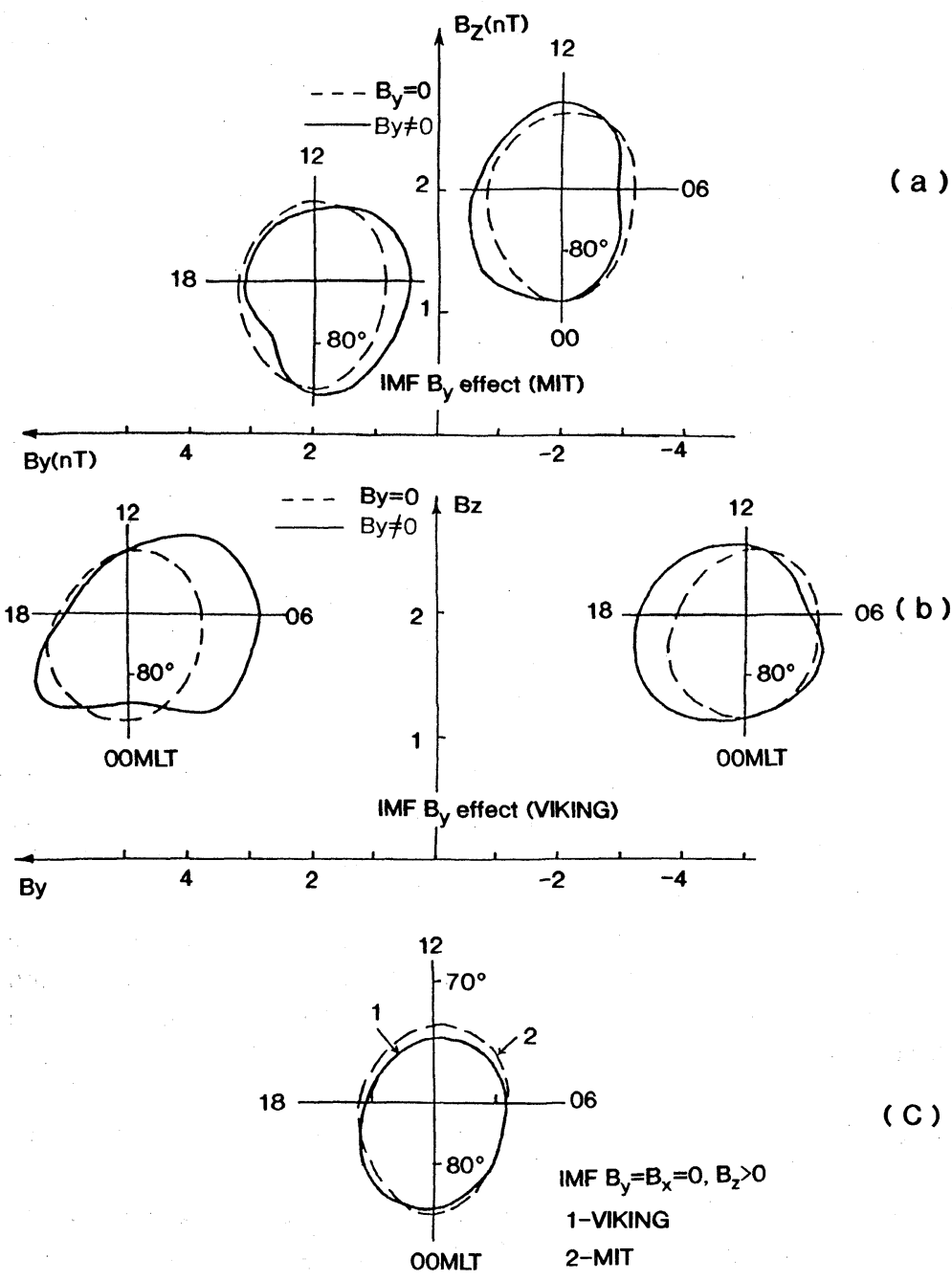

Fig. 2. Polar cap boundary dependence on the IMF $B_{y}$ component: (a) according to MIT-data, and (b) according to Viking imager data (ELPHINSTONE et al., 1990). Dashed lines are the polar cap boundaries for IMF $B_{y}=0$. (c) Comparison of polar cap boundaries from MIT and Viking images for IMF $B_{y}=0$. 
typical (average) error of one instantaneous value was larger than 2.5 degrees of latitude. Therefore, the 8 "best events", one for each value of $\beta$, were selected from the above-mentioned 32 events which provided best viewing conditions. Two series of polar cap boundaries were obtained ( 8 "average boundaries" and 8 "best examples") for each $\beta$ value. Both of them show clear IMF $B_{y}$-effects in accordance, both, in qualitative and in quantitative sense, with expectations from dayside magnetopause reconnection models. For $B_{y}>0\left(B_{y}<0\right)$ the polar cap boundary is shifted toward dawn (dusk) from the $B_{y}=0$ position; the maximum shift is $\geq 5^{\circ}$ on the dawn-dusk meridian. This effect is expressed more systematically in the "best examples" than in the "average boundaries". Therefore, in Fig. 2(b) two boundaries from the "best examples" are shown for $B_{y}>0$ (average $\beta \approx 90^{\circ}$ ) and $B_{y}<0$ (average $\beta \approx 270^{\circ}$ ), respectively. Furthermore, the statistical (average) boundary (for $B_{y}=0$ ) is shown. The ELPHINSTONE et al. (1990) paper contained only signs of IMF $B_{z}$ and $B_{y}$ for the "best examples" cases, not absolute values, therefore in Fig. 2(b) arbitrary units for the IMF are used (in contrast to Fig. 2(a)). We will use these two "best examples" and the average boundary to test corresponding MIT outputs.

Polar cap boundaries were determined by MIT according to selection criteria similar to those described above (the IMF had to be stable for more than 20 to 30 min preceding the time interval under consideration). 17 events were selected. They are listed in Table 1 together with their characteristics. Average polar cap boundaries, calculated by MIT both for IMF $B_{y}>0$ and $B_{y}<0$, are shown in Fig. 2(a). The boundary for $B_{y}=0$ is also shown (dashed line). In Fig. 2(c) the two average polar cap boundaries for $B_{y}=0$ obtained from MIT and from Viking images are compared. It is easy to see that good qualitative agreement exists between the two methods for all three IMF orientations considered (for a more quantitative comparison, see Section 4).

A comparison was also made between polar cap boundaries calculated by MIT and those derived from the Tsyganenko 1987 model. Figure 3(a) shows MIT data for a time interval with $K p=0$ and for an interval with $A E=750 \mathrm{nT}$ (the latter is a CDAW 9C substorm interval; 3 May,

Table 1 .

\begin{tabular}{rrrrrrr}
\hline & Date & UT & Viking orbit & $A E(\mathrm{nT})$ & $B_{z}(\mathrm{nT})$ & $B_{y}(\mathrm{nT})$ \\
\hline 1 & 860925 & 1950 & 1188 & 190 & 3.6 & -1.7 \\
2 & & 2010 & & 160 & 2.5 & -1.0 \\
3 & & 2030 & & 150 & 2.5 & -1.0 \\
4 & & 2040 & & 130 & 2.5 & -1.0 \\
5 & & 2050 & & 190 & 2.5 & -1.0 \\
6 & 861110 & 1900 & 1441 & 60 & 0.9 & -1.6 \\
7 & & 1910 & & 60 & 1.0 & -2.0 \\
8 & & 1920 & & 60 & -0.2 & -0.4 \\
9 & & 1930 & & 60 & 1.5 & -2.0 \\
10 & & 1940 & & 80 & 1.1 & -3.0 \\
11 & & 1950 & & 100 & 0.8 & -3.9 \\
12 & 860411 & 0545 & 266 & 200 & 1.0 & 1.0 \\
13 & 860411 & 1035 & 267 & 80 & 0 & 1.0 \\
14 & 860415 & 1035 & 289 & 90 & -1.0 & 1.0 \\
15 & 860422 & 2230 & 336 & 70 & 5.0 & 6.5 \\
16 & 860518 & 0330 & 469 & 60 & 2.0 & 6.0 \\
17 & 860525 & 0110 & 507 & 90 & 0 & 3.0 \\
\hline
\end{tabular}



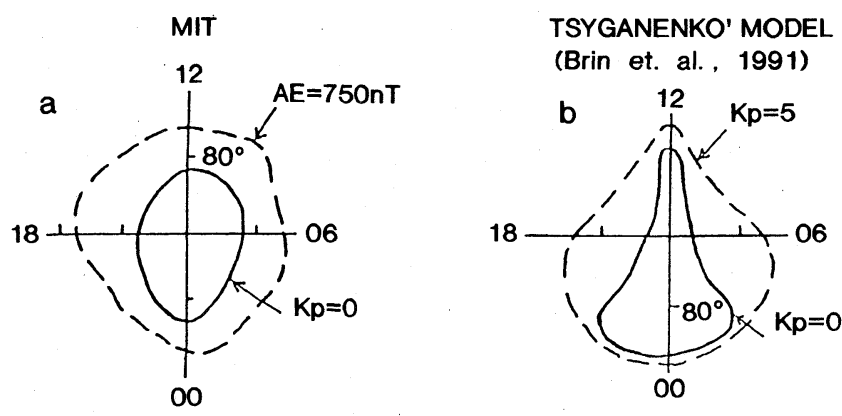

CDAW-9-C-substorm. AE $=750 \mathrm{nT}$

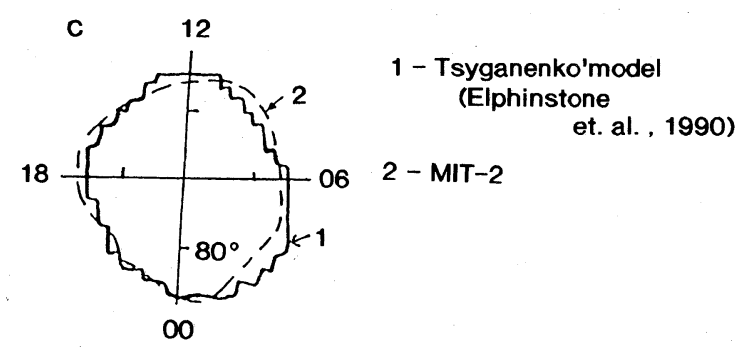

Fig. 3. Polar cap boundaries for quiet and disturbed conditions obtained from MIT for $K p=0$, and $A E=750 \mathrm{nT}$ (CDAW-9-C; 0945UT; 3 May, 1986) (a); and from mapping using the Tsyganenko 1987 model (b). (c) A comparison of polar cap boundaries obtained from MIT and from Tsyganenko model for 0945UT; 3 May, 1986 $(A E=750 \mathrm{nT})$.

1986; 0945 UT). Figure 3(b) shows polar cap boundaries for two comparable conditions obtained from the Tsyganenko model (BIRN et al., 1991). In Fig. 3(c) two polar cap boundaries, both for 0945 UT; 3 May, 1986, are shown; one is the MIT boundary, the other one is taken from ELPHINSTONE et al. (1991) and is based on mapping with the Tsyganenko 1987 long tail model. It is obvious that both methods yield a growth of the polar cap area with increasing activity by a factor of approximately 2.5. However, the horse collar shape of the quiet boundary as seen in Fig. 3(b), is not obtained with the MIT method (Fig. 3(a)).

\section{Discussion and Conclusions}

In Section 3 two independent methods have been used for a comparison with polar cap boundaries estimated with the MIT method. Qualitatively, a good agreement was obtained. The characteristic error of polar cap boundary determination from Viking auroral images is more than $2^{\circ}$ geomagnetic latitude (see Table 1 of ELPHINSTONE et al., 1990). The uncertainty of polar cap boundaries derived from the Tsyganenko magnetic field model is not less, since the Tsyganenko model is working rather crude in the region of interest. This implies that for IMF $B_{y}=0$ (Fig. 2(c)) deviations between polar cap boundaries derived from the MIT method and those using Viking images are within the uncertainties of the models. The same is true for MIT- and Tsyganenko model boundaries for $A E=750 \mathrm{nT}$ (Fig. 3(c)).

The disagreement between polar cap boundaries derived from MIT and those derived 
from Viking auroral images is larger than $2^{\circ}$ for IMF $B_{y}>0$ and $B_{y}<0$ conditions (Figs. 2 and $3)$. However, the error is still less than the IMF $B_{y}$ dependent shift and one has to bear in mind that the polar cap boundaries of Figs. 2(a) and 2(b) are derived using different time intervals and thus the deviations may be attributed to differences in the magnitude of the $B_{y}$ component in these intervals.

Figures 3(a) and 3(b) consistently show an increase of the polar cap area $S$ by a factor of approximately 2.5 going from $K p=0$ to $K p=5($ or $A E=750 \mathrm{nT})$ conditions. This result is rather important for substorm studies where the parameters $\Psi=S B o$ (the open tail magnetic flux) and $\varepsilon_{1}{ }^{\prime} \approx$ const $\Psi^{2}$ (the input power from the solar wind) both play a decisive role. The increase of $\Psi$ by a factor of $\leq 2$ during a substorm active phase following the growth phase is the major signature of a specific substorm phase, named the "1st active phase". In this signature the 1st active phase differs from the well-known expansion phase. For the latter, a decreasing of $\Psi$ is the characteristic signature. The two above mentioned active phases differ by major physical processes. The 1st active phase is characterized by tail stretching and energy transfer intensification as well as instabilities in the innermost part of the cross-tail current $\left(-x<8 \mathrm{R}_{\mathrm{E}}\right)$ without a neutral line formation; during an expansion phase the major process is the tail contraction as the result of the neutral line formation in the region $-x>10 \mathrm{R}_{\mathrm{E}}$ (e.g. MISHIN, 1991).

Thus, the agreement between the MIT (Fig. 3(a)) and the Tsyganenko magnetic field model (Fig. 3(b)) regarding the growth of the polar cap size with increasing disturbance level is essential. However, the disagreement between the shapes of the polar cap in Fig. 3(a) and those in Fig. 3(b) (in the latter a horse collar shape polar cap is seen) may be of importance and additional studies are desirable.

In summary, the work performed has shown that MIT provides reliable information about the dynamics of the polar cap boundaries and polar cap area, with an accuracy high enough, to study e.g. the open tail magnetic flux variations during substorms, as well as IMF-effects. This conclusion supports the major results obtained from substorm studies which are based on MIT.

The authors from SibIZMIR thank The Royal Swedish Academy of Sciences and Prof. Bengt Hultqvist at the Swedish Institute of Space Physics for the stimulating discussions, financial support and hospitality during visits to Kiruna (Sweden), where much of this work was carried out. The Viking project was managed by the Swedish Space Corporation and financed by the Swedish National Space Board.

\section{REFERENCES}

Birn, J., E. W. Hones, Jr., J. D. Craven, L. A. Frank, R. D. Elphinstone, and D. P. Stern, On open and closed field line regions in Tsyganenko's field model and their possible associations with horse collar auroras, J. Geophys. Res., 96, 3811-3817, 1991.

COLEY, W. R., Spatial relationship of field-aligned currents, electron precipitation, and plasma convection in the auroral oval, J. Geophys. Res., 88, 7131-7141, 1983.

COWLEY, S. W. H., Magnetospheric asymmetries associated with the $y$-component of the IMF, Planet. Space Sci., 29, 79, 1981.

Elphinstone, R. D., K. Jankowska, J. S. Murphree, and L. L. Cogger, The configuration of the auroral distribution for interplanetary magnetic field $B_{z}$ northward 1. IMF $B_{x}$ and $B_{y}$ dependencies as observed by the Viking satellite, J. Geophys. Res., 95, 5791-5804, 1990.

Elphinstone, R. D., D. Hearn, J. S. Murphree, and L. L. Cogger, Mapping using the Tsyganenko long magnetospheric model and its relationship to Viking auroral images, J. Geophys. Res., 96, 1467-1480, 1991. 
Gussenhoven, M. S., D. A. HARdy, N. Heinemann, and R. K. Burkhardt, Morphology of the polar rain, J. Geophys. Res., 89, 9785-9800, 1984.

HAREl, M., R. A. Wolf, P. H. Reiff, R. W. SPIRo, M. J. BurCE, F. Rich, and M. SMidDy, Quantitative simulation of a magnetospheric substorm 1. Model logic and overview, J. Geophys. Res., 86, 2217, 1981.

IIJIMA, T. and T. A. PotemRA, Field-aligned currents in the dayside cusp observed by TRIAD, J. Geophys. Res., 81, 5971-5979, 1976.

LASSEN, K. and C. DANIELSEN, Distribution of auroral arcs during quiet geomagnetic conditions, J. Geophys. Res., 94, 2587-2594, 1989.

Lockwood, M., The excitation of ionospheric convection, J. Atm. Terrestr. Phys., 53, 177, 1991.

MAKITA, K. and C.-I. MENG, Average electron precipitation patterns and visual aurora characteristics during geomagnetic quiescence, J. Geophys. Res., 89, 2861-2872, 1984.

MAKITA, K., C.-I. MENG, and S.-I. AKASOFU, The shift of the auroral electron precipitation boundaries in the dawndusk sector in association with geomagnetic activity and interplanetary magnetic field, J. Geophys. Res., 88, 7967-7981, 1983.

MAKITA, K., C.-I. MENG, and S.-I. AKASOFU, Latitudinal electron precipitation patterns during large and small IMF magnitudes for northward IMF conditions, J. Geophys. Res., 93, 97-104, 1988.

MENG, C.-I., in Electromagnetic Coupling in the Polar Clefts and Caps, edited by P. E. Sandholt and A. Egeland, p. 61, Kluwer Academic, Boston, Mass., 1989.

Mishin, V. M., The magnetogram inversion technique and some applications, Space Sci. Rev., 53, 83-163, 1990.

Mishin, V. M., The magnetogram inversion technique: applications to the problem of magnetospheric substorms, Space Sci. Rev., 57, 237-337, 1991.

REIFF, P. H. and J. L. BURCH, IMF By-dependent plasma flow and Birkeland currents in the dayside magnetosphere, 2. A global model for northward and southward IMF, J. Geophys. Res., 90, 1595, 1985. 